 \\ www4.fsanet.com.br/revista
}

Rev. FSA, Teresina, v. 18, n. 11, art. 3, p. 44-67, nov. 2021

ISSN Impresso: 1806-6356 ISSN Eletrônico: 2317-2983

http://dx.doi.org/10.12819/2021.18.11.3

\section{Análise do Mercado de Criptomoedas Sob o Enfoque da Matriz Swot}

\section{Analysis of the Crypto Currency Market Under the Swot Matrix Perspective}

Paulo Guerreiro Braga Júnior Graduação em Administração pela Universidade Federal do Rio de Janeiro paulogbjr@hotmail.com

Boris Asrilhant

Doutor em Industrial and Business Studies University of Warwick Professor Adjunto da Faculdade de Administração e Ciências Contábeis da UFRJ boris@facc.ufrj.br

\section{Endereço: Paulo Guerreiro Braga Júnior}

Av. Pedro Calmon, 550 - Cidade Universitária da Universidade Federal do Rio de Janeiro, Rio de Janeiro RJ, 21941-901, Brasil.

Endereço: Boris Asrilhant

Av. Pedro Calmon, 550 - Cidade Universitária da Universidade Federal do Rio de Janeiro, Rio de Janeiro RJ, 21941-901, Brasil.
Editor-Chefe: Dr. Tonny Kerley de Alencar Rodrigues

Artigo recebido em 31/10/2021. Última versão recebida em 10/10/2021. Aprovado em 11/10/2021.

Avaliado pelo sistema Triple Review: a) Desk Review pelo Editor-Chefe; e b) Double Blind Review (avaliação cega por dois avaliadores da área).

Revisão: Gramatical, Normativa e de Formatação 


\title{
RESUMO
}

Este artigo apresenta uma análise do mercado de criptomoedas, sob o enfoque da Matriz SWOT, a partir da identificação e correlação entre as forças e fraquezas do ambiente interno e as oportunidades e ameaças do ambiente externo. As informações foram coletadas através de uma extensa pesquisa bibliográfica sobre Blockchain e o mercado de criptomoedas, complementada por uma pesquisa documental pública atualizada, com base nas ideias preconizadas pela Análise de Conteúdo de Bardin, cujo critério adotado foi coletar tópicos citados por mais de uma fonte, demonstrando, assim, a sua relevância. Foram selecionadas quatro forças, quatro fraquezas, quatro oportunidades e quatro ameaças, que formaram a Matriz SWOT do Mercado de Criptomoedas. Em seguida, foram correlacionadas as variáveis do ambiente interno com as variáveis do ambiente externo, sendo validadas onze correlações denominadas de Alavanca (Força x Oportunidade), Defesa (Força x Ameaça), Restrição (Fraqueza x Oportunidade) e Problema (Fraqueza x Ameaça). A partir da análise destas correlações foram apresentadas as conclusões deste trabalho, dentre elas destacam-se que o mercado de criptomoedas é um investimento promissor com aplicação multifuncional para a sociedade, mas ainda apresenta elevados riscos devido ao seu atual nível de amadurecimento.

Palavras-Chave: Criptomoedas. Blockchain. Bitcoin. Matriz SWOT.

\begin{abstract}
This article presents an analysis of the crypto currency market under the SWOT Matrix perspective, considering the identification and correlation among the internal environment strengths and weaknesses and the external environment opportunities and threats. The data gathering included an extensive bibliographic research regarding Blockchain and the crypto currency market, complemented by an updated public document research based on the ideas presented by Bardin's Content Analysis, whose adopted criterion was to collect topics cited by more than one source in order to ratify its relevance. Four strengths, four weaknesses, four opportunities and four threats were selected to create the Crypto Currency Market SWOT Matrix. Then, the internal environment variables were correlated with the external environment ones, being eleven correlations considered valid, named Leverage (Strength $x$ Opportunity), Defense (Strength x Threat), Restriction (Weakness x Opportunity) and Problem (Weakness $\mathrm{x}$ Threat). Based on the correlations analysis, conclusions were presented, among them one highlights that the crypto currency market is a promising investment with multifunction application for society, but still presents some high risks due to its present maturation level.
\end{abstract}

Keywords: Crypto currency market. Blockchain. Bitcoin. SWOT Matrix. 


\section{INTRODUÇÃO}

Em 2008 ocorreu uma das piores crises econômicas mundiais. O sistema financeiro dos Estados Unidos colapsou devido a uma bolha imobiliária, por conta de uma política de crédito arriscada, onde o aumento nos valores imobiliários não foi acompanhado pelo aumento de renda da população (FREITAS, 2020). Segundo dados obtidos em 2013 pela Edelman Trust Barometer, a confiança das populações americana e europeia em relação ao sistema financeiro bancário caiu consideravelmente durante o período de 2008 a 2013, ficando abaixo da linha de confiança de 50\% (EDELMAN, 2013).

A partir desse contexto, a ideia de criação do Bitcoin começa a ganhar mais apoio. Através do Blockchain, um sistema peer-to-peer de dinheiro eletrônico, permitiu-se que pagamentos on-line fossem enviados diretamente de uma parte para outra, sem passar por uma instituição financeira (NAKAMOTO, 2008).

A inovação que desafiava os bancos, criada por Satoshi Nakamoto, revolucionou as transações financeiras. O Bitcoin, que utiliza a versão Blockchain 1.0, é visto atualmente como uma reserva eficiente de valor, já que é anti-inflacionária por design (HARADA, 2020).

As criptomoedas alternativas ao Bitcoin, chamadas de altcoins, também ganharam seu espaço no mercado, que é o caso do Ethereum. As altcoins proporcionaram a versão Blockchain 2.0, que possibilita o uso de contratos inteligentes, isto é, acordos de valor legal entre partes sem a necessidade de um intermediário. Ainda existe a versão Blockchain 3.0, que amplia a utilidade desta tecnologia para mais serviços (SWAN, 2015).

Tais fatores impulsionaram o crescimento exponencial do mercado de criptomoedas. Em maio de 2013, a capitalização de mercado somava aproximadamente 2,1 bilhões de reais. Já em agosto de 2021, esse número era cerca de 8,12 trilhões de reais (COINMARKETCAP, 2021), um aumento de aproximadamente $4.000 \%$ em oito anos.

Entretanto, trata-se de um mercado novo que ainda carece de regulamentação contra manipulações e fraudes, assuntos que ainda são muito discutidos no âmbito jurídico. $\mathrm{O}$ aparecimento de criptomoedas falsas, esquemas de pirâmide utilizando o Bitcoin como moeda de referência e casos de lavagem de dinheiro através de criptomoedas provocaram desconfiança de muitos investidores em relação à segurança desse mercado.

Além disso, os governos também podem interferir no uso de criptomoedas, como o exemplo do caso da Rússia, que, por meio de força de lei, ficaram proibidos os pagamentos através de criptoativos (THE BLOCK, 2020). 
Portanto, este artigo visa compreender os principais conceitos do Blockchain, bem como analisar as principais características e a dinâmica do mercado de criptomoedas, ainda bastante recente e com muitos prós e contras. Para tal, será proposta a matriz SWOT do mercado de criptomoedas e serão analisadas as correlações entre as suas variáveis.

\section{REFERENCIAL TEÓRICO}

\subsection{O Conceito de Blockchain}

Blockchain é uma tecnologia de gerenciamento de dados que funciona como uma rede de transações de criptoativos entre indivíduos, sem a assistência de intermediários (MOUGAYAR, 2017). Seu conceito surgiu em 2008, no artigo "Bitcoin: Um sistema financeiro eletrônico peer-to-peer”, publicado por uma pessoa ou grupo sob o pseudônimo de Satoshi Nakamoto (PRADO, 2018).

De acordo com Swan (2015), as diferentes atividades do Blockchain são divididas em três categorias: Blockchain 1.0, 2.0 e 3.0. O Blockchain 1.0, diretamente relacionado ao Bitcoin, apresenta uma grande importância na criação do conceito de criptomoedas virtuais e no sistema de pagamento digital (SILVA; BOVÉRIO, 2018).

Todo indivíduo que negocia criptomoedas possui uma carteira digital. Por meio de uma operação criptográfica, a carteira é capaz de gerar diversos endereços que consistem em uma combinação de caracteres e números. Para qualquer transação de criptomoedas é necessário que o destinatário informe o endereço ao remetente (BORTOLINI, 2018).

A validação das transações acontece através de empresas com alto poder computacional, especializadas em mineração de criptomoedas. Depois de uma competição matemática entre as máquinas, o computador vencedor recebe o direito da verificação, cujos proprietários recebem uma pequena quantia de criptomoeda como recompensa pela conclusão do processo, chamada de Proof of Work.

As transações do Blockchain são armazenadas no ledger, que é considerado um livro digital imutável, cuja função é registrar informações visíveis para todos (FERREIRA et al., 2017). O fato de o Blockchain ser transparente, auditável e consultável é ressaltado por Sobrinho et al. (2019).

Para impedir que o ledger seja modificado e garantir a segurança do Blockchain, é aplicado o conceito de hash, que é uma função matemática que gera um código alfanumérico através dos dados inseridos (PRADO, 2018). Portanto, para que uma fraude seja bem- 
sucedida, é necessária uma engenharia de processamento computacional muito sofisticada para o recálculo de todos os hash existentes (BORTOLINI, 2018).

O processo de mineração no Blockchain, por sua vez, gera um altíssimo consumo de energia, que resulta em um impacto desfavorável ao meio ambiente (SILVA; RODRIGUES, 2017). Em 2014, a rede de computadores utilizada para produzir criptomoedas em escala planetária consumiu o equivalente à energia elétrica gasta pela Irlanda (PIRES, 2016).

O Blockchain 2.0, iniciado pela criptomoeda Ethereum, é marcado pela introdução do conceito de contratos inteligentes, ou seja, qualquer contrato que formalize negociações entre partes sem a necessidade de intermediários (CARDOSO, 2018). Braga et al. (2017) citam a aplicabilidade do Blockchain 2.0 em diferentes mercados, como o de telecomunicações, onde através dos contratos inteligentes as operadoras conseguem cortar custos e oferecer serviços digitais a preços mais competitivos.

Já o Blockchain 3.0 envolve operações para toda a sociedade (BELMIRO, 2018). Para Swan (2015), a versão 3.0 apresenta aplicações eficientes, coordenadas e multifuncionais, atuando, segundo Silva e Bovério (2018), como protagonista na revolução de distribuição de dados.

Assim sendo, o Blockchain é uma tecnologia disruptiva que apresenta diversas funcionalidades para a sociedade, pautada pela transparência, segurança e privacidade. Entretanto, a sua eficiência está diretamente ligada à atualização e ao desempenho da adoção em massa da rede.

\subsection{Mercado de Criptomoedas}

O mercado de criptomoedas iniciou-se em 2009, com a criação do Bitcoin. As criptomoedas possuem, como principal finalidade, serem reservas de valor, oferecendo proteção do patrimônio contra variações e imprevisibilidade do mercado, e moedas de troca, podendo ser utilizadas para compra e venda de bens e serviços (MOUGAYAR, 2017).

Atualmente, o mercado é divido em duas classes de ativos: Bitcoin, a principal e mais antiga criptomoeda do mercado e as Altcoins, que são criptomoedas com diferentes características e finalidades.

O Bitcoin é divisível e portátil, porém não funciona exclusivamente como uma moeda digital, mas como um sistema de pagamento global online e descentralizado (ARAÚJO; SILVA, 2017). Segundo Santos (2016), a característica descentralizadora faz com que o sistema das criptomoedas se diferencie do sistema bancário convencional. 
As altcoins são uma classe de ativo alternativa no mercado de criptomoedas. Essas moedas possuem projetos próprios, que visam aprimorar ou criar serviços mais eficientes em beneficio da população. Criada em 2016, a altcoin mais popular do mercado é o Ethereum, que introduziu a tecnologia de contratos inteligentes e possui forte investimento da IBM e Microsoft (ARAÚJO; SILVA, 2017).

Cabe ressaltar que as criptomoedas são emitidas gradativamente para que não ocorra a sua desvalorização, assim, conforme os indivíduos trocam suas moedas por Bitcoins, novas moedas serão liberadas até o novo total disponível (CAMPOS, 2015). Portanto, o Bitcoin é deflacionário por natureza. O número limitado do ativo associado à sua crescente adoção implica o aumento do seu poder de compra ao longo do tempo (SOUSA, 2021). Alexandre (2020) reforça a característica deflacionária ao citar que esta criptomoeda possui emissão controlada em níveis decrescentes.

A população norte-americana é a que mais negocia criptomoedas no mundo (ANDRADE, 2021) e o ambiente externo atual, de inflação crescente, favorece esse fato, já que as criptomoedas têm caráter deflacionário. Como forma de amenizar os danos econômicos causados pelo COVID-19, apenas em 2020, o Banco Central dos Estados Unidos (Federal Reserve), emitiu 22\% de toda a oferta de dólares disponíveis no mercado (MARINHO, 2020). Tal medida impactou diretamente a inflação dos Estados Unidos, que atingiu o maior nível em treze anos (HESSEL, 2021).

Os Estados Unidos não são o único país afetado com inflação crescente. Em um ranking de dados de inflação de países da América Latina, em um período de 12 meses até julho de 2021, elaborado pelo Instituto Brasileiro de Economia da Fundação Getúlio Vargas, há países com índice de inflação anual elevada como, por exemplo, Argentina, que somou 51,8\%, Haiti, 17,9\% e Brasil com 9,0\% (LIMA; GERBELLI, 2021).

Além disso, a implementação do Bitcoin como moeda legal possui o potencial de melhorar a qualidade de vida daqueles com acesso restrito a serviços financeiros, principalmente nas regiões mais pobres (ULRICH, 2014). Todos podem negociar Bitcoin através de um aparelho eletrônico, como o celular, sem uma instituição intermediária.

Segundo Dow (1982), as condições econômicas predominantes nas regiões periféricas implicam em níveis de incerteza maiores percebidos por parte das instituições financeiras. Portanto, segundo Fialho et al. (2016), essas regiões possuem limite de crédito, menos oportunidades de investimento e são mais restritas a depósitos à vista. Esses fatores podem influenciar a vulnerabilidade dos locais menos desenvolvidos, os tornando mais dependentes das regiões centrais. 
Para Campos (2015), não é necessário que se crie uma legislação rígida sobre as criptomoedas, pois há o risco de limitar a nova tecnologia. Entretanto, o Estado deve buscar a proteção dos indivíduos que utilizam criptomoedas. A carência e a dificuldade de regulamentação desse segmento de mercado também induzem à sua aversão pelas grandes instituições financeiras, que não conseguem acompanhar a velocidade das novas situações criadas em um espaço desprovido de normas, que é o meio digital (SICHEL; CALIXTO, 2021).

As criptomoedas apresentam variações significativas nos preços, o que não é usual para ativos tradicionais (CIAIAN et al., 2016). Essa alta volatilidade é uma das principais características que devem ser consideradas antes de começar a investir (ULRICH, 2014).

Mesmo assim, o mercado de criptomoedas está em pleno crescimento. O surgimento de criptomoedas com projetos promissores para otimização de serviços aliados a parcerias com empresas podem ser fundamentais para que, em breve, as criptomoedas substituam diversos serviços ineficientes para população (ARAÚJO; SILVA, 2017). Entretanto, trata-se de um mercado em processo de amadurecimento, exigindo cautela por parte dos investidores.

\section{METODOLOGIA}

Segundo Vergara (1998), a pesquisa adotada por este trabalho é tanto descritiva, pois apresenta as principais características do Blockchain e do mercado de criptomoedas, quanto exploratória, pois, segundo Mattar (1994), a pesquisa exploratória visa prover o pesquisador de um maior conhecimento sobre um determinado tema.

A coleta de dados será feita através de duas etapas, uma pesquisa bibliográfica baseada em publicações acadêmicas, complementada por uma pesquisa documental na internet, de modo a inserir informações mais atualizadas, dado o dinamismo do tema.

A coleta de dados será realizada sob dois enfoques: a ótica da matriz SWOT e as ideias preconizadas pela Análise de Conteúdo, conforme apresentada a seguir.

A análise SWOT, criada por Kenneth Andrews e Roland Christensen (PONTES, 2014), visa analisar os ambientes interno e externo de uma organização ou setor. A sigla SWOT, segundo Carneiro (2010), envolve Forças (Strengths), ou seja, fatores identificados no ambiente interno que trazem um diferencial e/ou uma vantagem competitiva; Fraquezas (Weaknesses), que são limitações ou ineficiências no ambiente interno que podem restringir o desempenho; Oportunidades (Opportunities), sendo as situações do ambiente externo 
benéficas ao sucesso; e Ameaças (Threats), situações do ambiente externo que podem ser prejudiciais ao sucesso.

A partir da proposição da matriz SWOT do mercado de criptomoedas, serão analisadas as correlações das forças e fraquezas com as oportunidades e ameaças (CHIAVENATO; SAPIRO, 2003). Essas correlações apresentam quatro tipos de situações: Forças $\mathrm{x}$ Oportunidades (Alavanca), quando há oportunidades externas compatíveis com os pontos fortes e por isso é possível extrair o máximo de proveito da situação; Forças x Ameaças (Defesa), quando existem ameaças amenizadas pelos pontos fortes; Fraquezas $\mathrm{x}$ Oportunidades (Restrição), quando há oportunidades que não podem ser aproveitadas devido aos pontos fracos; e Fraquezas x Ameaças (Problema), quando as ameaças são potencializadas devido aos pontos fracos (DAYCHOUM, 2007).

O segundo enfoque adotado utilizará as ideias apresentadas na Análise de Conteúdo (Bardin, 2011). A Análise de Conteúdo não se limita à descrição dos dados coletados, mas às inferências obtidas sobre estes dados, de modo a validar uma determinada proposição, ainda que seja proposta por visões diversas dentro de um mesmo contexto.

Assim sendo, no processo de seleção dos elementos dos principais elementos que compõem a matriz SWOT do mercado de criptomoedas, foi estabelecido um critério objetivo para validação desse processo, através da citação por mais de uma fonte, caracterizando, assim, a sua importância.

\subsection{Pesquisa Documental sobre Blockchain e Mercado de Criptomoedas}

De acordo com o site CoinMarketCap (2021), já existem cerca de 13.000 criptomoedas no mercado. As três criptomoedas que possuem maior valor de mercado são Bitcoin, Ethereum e Binance Coin, nesta ordem. O valor total do mercado de criptomoedas é de aproximadamente 2,6 trilhões de dólares, sendo cerca de $45 \%$ desse valor dominado pelo Bitcoin.

O Bitcoin foi a primeira criptomoeda criada no mundo, o que explica sua dominância do mercado. Seu Blockchain é conhecido pela alta segurança, já que nunca foi invadido. Fato este que não se aplica ao Blockchain de todas as criptomoedas, que já apresentaram erros ou bugs que causaram insegurança aos investidores, como por exemplo, quando o Blockchain da Poly Network apresentou um erro e sofreu um roubo por hackers de aproximadamente US\$ 600 milhões (TIDY, 2021). A rede Solana também já apresentou bug em sua rede Blockchain, quando teve que reiniciá-la para solucionar um bug crítico, acarretando em um período de mais de 16 horas sem transações financeiras (ALVES, 2021). Aliado ao problema de bugs, 
segundo Nascimento (2021), as atualizações dos sistemas depende da tecnologia disponível. Varoni (2017) ressalta esse aspecto e afirma que a manutenção do Blockchain 3.0 é mais rápida do que das redes Blockchain 1.0 e 2.0.

A alta volatilidade dos preços é uma característica típica do mercado de criptomoedas, que pode ser visto como uma forma de alavancagem financeira, já que no período entre 2015 e 2021 o Bitcoin valorizou aproximadamente $20.000 \%$ e a criptomoeda Ethereum valorizou cerca de $45.000 \%$ (TRADINGVIEW, 2021).

$\mathrm{O}$ avanço do mercado de moedas digitais fez com que algumas renomadas instituições financeiras aumentassem sua exposição ao Bitcoin, como é o caso do Morgan Stanley, que oferece investimentos em criptomoedas e realiza periodicamente relatórios de análise (CRIPTONIZANDO, 2021).

Entretanto, existem bancos e países que possuem posições inflexíveis em relação às criptomoedas, reprimindo o seu uso. De acordo com Stein (2021), existem pelo menos onze países que baniram a negociação de criptomoedas, em um deles houve a condenação de indivíduos à prisão. Em janeiro de 2021, Agustín Carstens, gerente do Banco de Pagamentos Internacionais (BIS), declarou: "Os investidores devem estar cientes de que o Bitcoin pode muito bem colapsar por completo" (MESQUITA, 2021).

O gasto energético derivado do processo de mineração de dados é um fator limitante ao crescimento do mercado de criptomoedas. Em 2021, a multinacional Tesla, empresa automotiva sustentável, abdicou da ideia de aceitar Bitcoin como meio de pagamento, com a justificativa da poluição causada pelo uso de combustíveis fósseis no processo de mineração (PAYÀ, 2021). Em uma entrevista, Bill Gates, fundador da Microsoft e investidor de empresas sustentáveis, afirmou que se preocupa com o consumo de energia e a poluição causada pela mineração do Bitcoin (NEW YORK TIMES, 2021). De acordo com o Cambridge Bitcoin Eletricity em 2021, a mineração do Bitcoin já ocupa o $30^{\circ}$ lugar no ranking de consumo energético do planeta (DA REDAÇÃO, 2021).

O mercado de criptomoedas vem apresentando novidades em relação à sua funcionalidade. Em 2021 foram popularizados os NFTs (Non-Fungible Tokens), que são ativos digitais únicos e não replicáveis. Adotado principalmente pelos colecionadores de artes, os NFTs servem como registro de propriedade e autenticidade de um objeto digital em uma Blockchain (REDAÇÃO, 2021). Outro nicho em que o mercado de criptomoedas está adentrando é o de esportes, através de Fan Tokens, que são ativos digitais criados pelos clubes de futebol, onde o torcedor adquire direitos de votação nas decisões internas dos clubes (MERCADO BITCOIN, 2020). 
Em abril de 2021, a altcoin Cardano anunciou uma parceria com o governo da Etiópia, com o objetivo de digitalizar o sistema educacional no país, trazendo mais transparência ao processo de classificação das universidades, bem como evitando a falsificação de documentos e a adulteração de notas (CRIPTONIZANDO, 2021).

O mercado de criptomoedas, por sua vez, ainda é muito pequeno comparado aos mercados financeiros tradicionais, o que facilita a manipulação dos preços através da disseminação de notícias prejudiciais aos investidores (TRADINGVIEW, 2021).

Elon Musk já foi acusado de manipulação do mercado e criticado por exercer sua influência de forma indevida, devido ao episódio do seu tweet de apoio à criptomoeda Dogecoin, que gerou uma grande repercussão e supervalorizou a criptomoeda em 24 horas, se tornando a quinta criptomoeda de maior valor de mercado em abril de 2021 (IGNACIO, 2021). A empresa proprietária da criptomoeda Litecoin está sob investigação pelas autoridades americanas após emitir um falso anúncio de que seria feita uma parceria com a Walmart em setembro de 2021. Após o anúncio, a criptomoeda valorizou 30\% em poucos minutos, mas sua desvalorização ocorreu na mesma intensidade após a Walmart ter desmentido a notícia (BRANCO, 2021).

Em agosto de 2021 ocorreu a maior apreensão de criptomoedas da história do Brasil, cerca de $\mathrm{R} \$ 150$ milhões em criptoativos associados à empresa GAS Consultoria Bitcoin, que movimentou R $\$ 38$ bilhões em seis anos, sem que tenham sido aplicados (SADOK, 2021).

Além disso, o mercado de criptomoedas possui grande presença de scams, termo relacionado às criptomoedas falsas ou inexistentes. Um exemplo famoso de scam foi a criptomoeda One Coin, criada através da ideia que seria melhor que o Bitcoin. Porém, o projeto nunca foi concretizado e seus fundadores desapareceram após conseguirem grandes aportes de investidores (BERTOLUCCI, 2021).

Portanto, apesar de o mercado de criptomoedas estar se mostrando cada vez mais um investimento promissor e com tecnologia multifuncional, é fundamental atentar para os riscos que devem sempre ser levados em consideração pelo investidor.

\section{RESULTADOS E DISUSSÕES}

\subsection{Matriz SWOT das Criptomoedas}

A partir do descrito na seção de Metodologia, serão apresentados e justificados pelo número de citações, a seguir, os tópicos que farão parte da Matriz SWOT de criptomoedas. 


\subsubsection{Forças:}

1) Sistema de pagamento global descentralizado: Citado no referencial teórico por Santos (2016) e Araujo e Silva (2017).

2) Todas as informações do Blockchain abertas e consultáveis: Mencionado no referencial teórico por Ferreira et al. (2017) e Sobrinho et al. (2019).

3) Criptomoedas deflacionárias: Citado no referencial teórico por Campos (2015), Alexandre (2020) e Sousa (2021).

4) Utilização da tecnologia das criptomoedas em múltiplos segmentos: Mencionado no referencial teórico, por Swan (2015), Belmiro (2018) e Silva e Bovério (2018). Na pesquisa documental foi citada a notícia da Criptonizando (2021) sobre a parceria da Altcoin Cardano com o governo da Etiópia.

\subsubsection{Fraquezas:}

1) Alto consumo energético nas minerações: Citado por Pires (2016) e Silva e Rodrigues (2017) no referencial teórico e por Da Redação (2021) na pesquisa documental.

2) Alta volatilidade: Citado no referencial teórico por Ulrich (2014) e Ciaian et al. (2016). Na pesquisa documental, citado nas estatísticas divulgadas no site TradingView (2021).

3) Lentidão de atualizações do Blockchain 1.0 e 2.0: Apresentado no referencial teórico por Varoni (2017) e Nascimento (2021).

4) Dificuldade de regulamentação: Mencionado no referencial teórico por Campos (2015) e Sichel e Calixto (2018).

\subsubsection{Oportunidades}

1) Serviços Financeiros limitados em áreas mais pobres: Citado no referencial teórico por Dow (1982), Ulrich (2014) e Fialho et al. (2016), além de uma pesquisa divulgada pelo The Global Findex Database (ACUANT, 2020).

2) Diferentes mercados a serem explorados e otimizados: Apresentado no referencial teórico por Braga et al. (2017) e Cardoso (2018). Na pesquisa documental, é citado com a criação de Fan Tokens para os esportes e o surgimento de NFTs (MERCADO BITCOIN, 2020 e REDAÇÃO, 2021). 
3) Cenário de alta inflação de países: Mencionado na pesquisa documental por Hessel (2021) e Lima e Gerbelli (2021). Apesar de o cenário de alta inflação ser negativo para a economia de um país, as criptomoedas, sendo deflacionárias por design, podem ser consideradas uma opção de investimento neste cenário.

4) Adoção de grandes empresas sustentáveis: Citado na pesquisa documental através do episódio da Tesla relatado por Payà (2021) e da entrevista de Bill Gates (New York Times, 2021).

\subsubsection{Ameaças}

1) Repressão de países a criptomoedas: Citado na pesquisa documental com a lista de países que baniram as criptomoedas apresentada por Stein (2021), e o discurso feito por Agustín Carstens, citado por Mesquita (2021).

2) Crimes através de criptomoedas: Citado na pesquisa documental pelas reportagens referentes à GAS Consultoria Bitcoin (SADOK, 2021) e à criptomoeda One Coin (BERTOLUCCI, 2021).

3) Influências e Manipulações no mercado: Apresentado através de reportagens feitas por Branco (2021) ao Canal Tech e por Ignacio (2021) ao Tecnoblog.

4) Erros ou bugs no Blockchain: Citado na pesquisa documental nas reportagens de Tidy (2021) e Alves (2021).

A Figura 1, a seguir, propõe a Matriz SWOT do Mercado de Criptomoedas. 
Figura 1 - Matriz SWOT das Criptomoedas

\begin{tabular}{|c|c|}
\hline $\begin{array}{l}\qquad \mathrm{FORC} \text { AS } \\
\text { - Sistema de pagamento global } \\
\text { descentralizado; } \\
\text { - Todas Informações do Blockchain } \\
\text { abertas e consultáveis; } \\
\text { - criptomoedas deflacionárias; } \\
\text { - Utilização da tecnologia das } \\
\text { criptomoedas em múltiplos } \\
\text { segmentos. }\end{array}$ & $\begin{array}{l}\quad \text { FRAQUF/AS } \\
\text { - Alto consumo energético nas } \\
\text { minerações; } \\
\text { - Alta volatilidade; } \\
\text { - Lentidão de atualizações do } \\
\text { Blockchain } 1.0 \text { e 2.0; } \\
\text { - Dificuldade de regulamentação. }\end{array}$ \\
\hline $\begin{array}{l}\text { OPORTUNID ADES } \\
\text { - serviços Financeiros limitados em } \\
\text { áreas mais pobres; } \\
\text { - Diferentes mercados a serem } \\
\text { explorados e otimizados; } \\
\text { - cenários de alta inflação de países; } \\
\text { - Adoção de grandes empresas } \\
\text { sustentáveis }\end{array}$ & $\begin{array}{l}\qquad \mathrm{AMEAC} A S \\
\text { - Repressão de países à } \\
\text { Criptomoedas; } \\
\text { - Crimes através de Criptomoedas; } \\
\text { - Influências e Manipulações } \\
\text { externas no Mercado; } \\
\text { - Erros ou bugs no Blockchain. }\end{array}$ \\
\hline
\end{tabular}

\subsection{Correlações da Matriz SWOT}

Com base na Matriz SWOT apresentada na Figura 1, foram analisadas onze correlações consideradas válidas, conforme apresentado na Figura 2 a seguir. 
Figura 2 - Correlações entre as Variáveis da Matriz SWOT das Criptomoedas

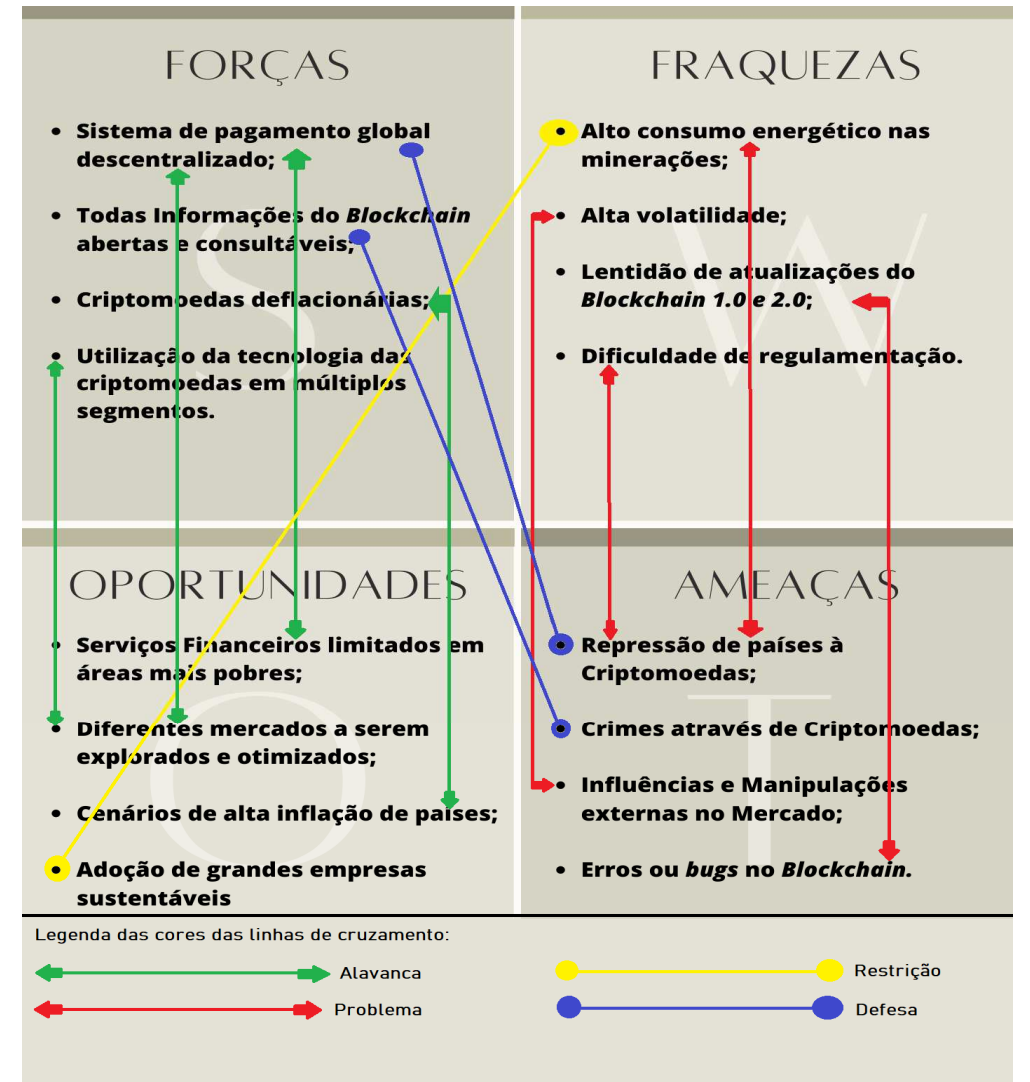

\subsubsection{Alavanca:}

1) Sistema de pagamento global descentralizado $X$ Serviços financeiros limitados em áreas pobres: As criptomoedas, em especial o Bitcoin, apresentam potencial para impactar positivamente a vida da população mundial e isso se deve à sua descentralização e globalização. Uma transação financeira de Bitcoin não precisa ser regulada por instituições financeiras, ou seja, sua característica descentralizadora pode ser crucial para aumentar o acesso financeiro em países ou áreas mais pobres, com menor acesso às instituições financeiras convencionais. Os dados do The Global Findex Database (ACUANT, 2020) mostram uma grande oportunidade para aumentar a adoção das criptomoedas por parte da população mundial. Apenas um celular já é suficiente para que o indivíduo realize transações financeiras com criptomoedas, já que em muitos países há mais pessoas com celulares do que com acesso a serviços financeiros básicos. Além disso, uma instituição financeira costuma avaliar se é rentável se estabelecer em áreas mais remotas, por ser, de acordo com Ulrich 
(2014), mais custoso. Já as criptomoedas, por serem ativos globalizados e que podem se beneficiar do cenário de desbancarização, não precisam fazer essa avaliação.

2) Sistema de pagamento global descentralizado $X$ Diferentes mercados a serem explorados e otimizados: Um sistema de pagamento global descentralizado pode ser útil na ampliação dos serviços de pagamentos. A indústria da música, citada por Cardoso (2018), é um exemplo, visto que a automação do processo através do Blockchain permitiu a distribuição dos royalties com a garantia da integridade financeira dos envolvidos, pelo fato de não existirem erros de precisão nos pagamentos.

3) Criptomoedas deflacionárias $X$ Cenário de alta inflação de países: $O$ Bitcoin é considerado deflacionário por ser um ativo que possui emissão controlada em níveis declinantes. Isso faz com que a criptomoeda possua um poder de compra maior com o passar do tempo, visto que, quanto menor a oferta de Bitcoins, maior será o seu preço. De acordo com um ranking elaborado em 2021 pela FGV sobre os dados de inflação de países da América Latina, trata-se de um cenário favorável para que os cidadãos dos países com maiores taxas de inflação considerem o uso de Bitcoin, ao invés de moedas que estão perdendo valor. A criptomoeda está imune à emissão desenfreada de moeda pelos governos e pode ser fundamental como hedge para a população durante as crises inflacionárias. Entretanto, deve ser levada em conta, nesta análise, a alta volatilidade das criptomoedas.

4) Utilização da tecnologia das criptomoedas em múltiplos segmentos $X$ Diferentes mercados a serem explorados e otimizados: A evolução da tecnologia do Blockchain está permitindo a inserção do mercado de criptomoedas em diversos setores da economia, como, por exemplo, no ramo dos esportes. Times populares, como PSG e Barcelona, foram pioneiros a oferecer Fan Tokens a fim de aprimorar os serviços aos torcedores. A multifuncionalidade do Blockchain vem despertando cada vez mais novos investidores interessados em soluções alternativas, o que faz aumentar a familiaridade dos indivíduos com o mercado de criptomoedas.

\subsubsection{Defesa}

1) Sistema de pagamento global descentralizado $X$ Repressão de países a Criptomoedas:

A falta de um agente intermediário para verificar as transações financeiras pode ser um problema sob a perspectiva governamental. Tal fato dá suporte à repressão de alguns países às criptomoedas. Entretanto, a proibição de países não interfere na negociação de criptomoedas entre indivíduos. De fato, um governo pode interferir nas compras de criptomoedas em 
exchanges e nas conversões pelas moedas físicas, mas a descentralização da rede limita as ações governamentais. Em setembro de 2021, o governo chinês proibiu todas as transações em criptomoedas no país e declarou que os ativos digitais eram ilegais (SÉRVIO, 2021). Mesmo após essa medida, houve um aumento significativo de transações de Bitcoin em ecossistemas de finanças descentralizadas (DeFi) provenientes do leste da Ásia (FINNESETH, 2021). Desde 2009, a China já determinou pelo menos cinco medidas restritivas à negociação de criptoativos (KALASHNIKOV, 2021). Tais acontecimentos reforçam o caráter defensivo da rede descentralizadora e a dificuldade dos países em proibirem as transações de criptomoedas.

\section{2) Todas as informações do Blockchain abertas e consultáveis $X$ Crimes através de}

Criptomoedas: Uma das maiores preocupações de novos investidores em relação ao mercado de criptomoedas é sobre a presença de crimes, especialmente a lavagem de dinheiro. $\mathrm{O}$ anonimato e a complexidade de rastreamento das transações no Blockchain atraem pessoas que desejam realizar esse tipo de crime, além das transações internacionais serem feitas de forma rápida, barata e sem a burocracia do modelo tradicional. Entretanto, o próprio Blockchain possui características que dificultam esses tipos de crime. Vale ressaltar que se trata de um livro digital imutável, onde todas as informações ficam armazenadas. Embora não apareçam os nomes dos participantes das transações financeiras, é possível rastrear pelo IP ou o endereço das carteiras digitais e mapear as ações realizadas. Em junho de 2021 foi noticiado que o FBI rastreou operação com Bitcoins no valor de aproximadamente US\$ 4,7 milhões, realizada por um ataque hacker a um oleoduto nos Estados Unidos (RUBINSTEINN, 2021). Mesmo sem divulgar como a investigação foi realizada, o acontecimento demonstra que não é tão simples promover atividades ilícitas com o uso de criptomoedas. De fato, as transações em Blockchain são mais complexas comparadas às transações financeiras tradicionais, ainda mais por se tratar de um ativo digital descentralizado. Portanto, os investigadores devem ser cada vez mais capacitados e providos de alto poder computacional para proteger o mercado de criptomoedas das atividades ilegais.

\subsubsection{Restrição}

1) Alto consumo energético nas minerações $X$ Adoção de grandes empresas sustentáveis:

A adoção por empresas sustentáveis é de extrema importância para ampliar o sucesso do mercado de criptomoedas. Entretanto, o ambiente da mineração é composto por uma grande concentração de máquinas ligadas durante 24 horas, que consomem muita energia, visto que 
devem ser mantidas à baixa temperatura para maximizar o seu desempenho. O Bitcoin já demonstrou ser frágil em relação à questão ambiental, quando a Tesla abdicou de aceitá-lo como forma de pagamento, o que ocasionou uma grande perda do seu valor de mercado Portanto, o alto consumo energético nas minerações pode ser uma barreira na oportunidade de adoção das criptomoedas por empresas sustentáveis.

\subsubsection{Problema}

1) Alto consumo energético $X$ Repressão de países a criptomoedas: A poluição e o alto consumo energético causado na mineração das criptomoedas são fatores desfavoráveis que podem influenciar a repressão de alguns países às criptomoedas. Em maio de 2021, o Irã baniu a mineração de criptomoedas por quatro meses a fim de evitar apagões no verão, que estavam sendo causados pelo grande consumo desta operação (SIMÕES, 2021). Portanto, episódios como esse aumentam a necessidade das empresas mineradoras de buscar, cada vez mais, fontes renováveis.

2) Alta volatilidade $X$ Influências e manipulações externas no mercado: A volatilidade do mercado de criptomoedas é totalmente diferente dos outros mercados de investimentos. Enquanto no mercado de ações uma queda ou alta de $5 \%$ em um ativo é considerada elevada, no mercado de criptomoedas é comum haver altas ou quedas de $20 \%$ em um dia, por exemplo. O mercado de criptomoedas ainda é recente e restrito, se comparado a outros mercados. A entrada de novos investidores de peso é fundamental para o seu processo de amadurecimento, de modo que o preço das criptomoedas seja cada vez menos vulnerável a notícias ou movimentações de compra, tais como boatos ou tweets informais publicados por grandes influenciadores. Esses acontecimentos são prejudiciais à credibilidade do mercado digital e podem criar uma barreira de entrada para novos investidores.

\section{3) Lentidão de atualizações no Blockchain 1.0 e 2.0 X Erros ou Bugs no Blockchain: A} atualização no sistema Blockchain é necessária para manutenção do ecossistema, porém o Blockchain 1.0 e 2.0 são mais lentos nesse quesito. Um grande intervalo de tempo entre as atualizações pode ser considerado um problema para os usuários das redes, visto que no mercado cibernético a otimização e a dinamicidade são cruciais. Os bugs ou erros aliados a essa demora podem gerar insegurança ainda maior em relação à usabilidade do Blockchain. Esse fato pode ser ainda mais preocupante para criptomoedas recentes que possuem uma reduzida equipe de desenvolvedores. Nesse caso, existe certa desconfiança do mercado em utilizar a rede desses protocolos no seu lançamento, por conta da possibilidade de bugs. 
4) Dificuldade de Regulamentação X Repressão de países a criptomoedas: A dificuldade de regulamentação no mercado de criptomoedas é um debate jurídico recorrente nos governos. Alguns países consideram que suas soberanias podem ser ameaçadas pelas criptomoedas, o que implica medidas radicais tomadas por estes governos. Acontecimentos, como de prisão de indivíduos que negociaram criptomoedas, podem provocar insegurança na entrada de novos investidores. $\mathrm{O}$ ideal seria que o debate de regulamentação fosse resolvido e que o conceito da moeda deflacionária por design fosse universalizado, o que seria benéfico para que os investidores se tornassem menos preocupados com a volatilidade causada por influência de agentes externos.

\section{CONSIDERAÇÕES FINAIS}

Este artigo apresentou uma análise do mercado de criptomoedas sob a ótica da Matriz SWOT, a partir do levantamento das forças e fraquezas, que compõem o ambiente interno, e das oportunidades e ameaças, que por sua vez compõem o ambiente externo.

Inicialmente, foi realizada uma pesquisa bibliográfica sobre Blockchain e o mercado de criptomoedas, que foi complementada por uma pesquisa documental pública na internet.

Para a criação da Matriz SWOT do Mercado de Criptomoedas foram utilizadas as ideias preconizadas pela Análise de Conteúdo concebida por Bardin (2011). Para tal, foi adotado o critério de citação por mais de uma fonte, de modo a validar a sua relevância.

A Matriz SWOT do Mercado de Criptomoedas foi formada pelos seguintes tópicos: Forças: Sistema de pagamento global descentralizado; Todas as informações do Blockchain abertas e consultáveis; Criptomoedas deflacionárias; Utilização da tecnologia das criptomoedas em múltiplos segmentos; Fraquezas: Alto consumo energético nas minerações; Alta Volatilidade; Lentidão de atualizações do Blockchain 1.0 e 2.0; Dificuldade de regulamentação; Oportunidades: Serviços financeiros limitados em áreas mais pobres; Diferentes mercados a serem explorados e otimizados; Cenários de alta inflação de países; Adoção de grandes empresas sustentáveis; e Ameaças: Repressão de países a criptomoedas; Crimes através de criptomoedas; Influências e manipulações externas no mercado; Erros ou bugs no Blockchain.

Em seguida, foram analisadas as correlações entre as variáveis do ambiente interno e as variáveis do ambiente externo. Onze correlações foram consideradas válidas, que são: Alavanca (Força X Oportunidade): Sistema de pagamento global descentralizado X Serviços financeiros limitados em áreas pobres; Sistema de pagamento global descentralizado $\mathrm{X}$ 
Diferentes mercados a serem explorados e otimizados; Criptomoedas Deflacionárias X Cenário de alta inflação de países; Utilização da tecnologia das criptomoedas em múltiplos segmentos X Diferentes mercados a serem explorados e otimizados; Defesa (Força X Ameaça): Sistema de pagamento global descentralizado $X$ Repressão de países à Criptomoedas; Todas as informações do Blockchain abertas e consultáveis X Crimes através de Criptomoedas; Restrição (Fraqueza X Oportunidade): Alto consumo energético nas minerações X Adoção de grandes empresas sustentáveis; e Problema (Fraqueza X Ameaça): Alto consumo energético $\mathrm{X}$ Repressão de países a criptomoedas; Alta volatilidade $\mathrm{X}$ Influências e manipulações externas no mercado; Lentidão de atualizações no Blockchain 1.0 e 2.0 X Erros ou bugs no Blockchain; Dificuldade de regulamentação X Repressão de países a criptomoedas

Uma primeira conclusão obtida da análise destas correlações foi que o mercado de criptomoedas já conseguiu superar diversas intervenções governamentais e, embora tenha sofrido momentaneamente, não houve dano permanente. Isso reforça que a sua característica descentralizadora é a força motriz deste ecossistema.

Além disso, trata-se de uma alternativa que pode ser útil para a otimização de diversos serviços e melhorar a qualidade de vida da população, especialmente dos mais pobres, com menos acesso a instituições financeiras tradicionais.

O cenário de alta inflação pode ser uma oportunidade para alavancar a adoção de criptomoedas por parte da população, por seu caráter deflacionário, já que as criptomoedas podem prevenir a diminuição do poder de compra dos seus investidores.

O alto consumo energético e a dificuldade de regulamentação são as fraquezas mais alarmantes, visto que ambas potencializam a ameaça mais impactante do ambiente externo, que é a repressão governamental à adoção de criptomoedas.

Por fim, o mercado de criptomoedas é um investimento promissor, visto que a multifuncionalidade do sistema alimenta perspectivas futuras favoráveis em diversos setores. Entretanto, o gerenciamento de risco do patrimônio é fundamental para mercados ainda em processo inicial de amadurecimento.

\section{REFERÊNCIAS}

ACUANT. The World's Unbanked Population. Acuant, 2020. Disponível em: < https://www.acuant.com/blog/the-worlds-unbanked-population/ > Acesso em 02 de setembro de 2021. 
ALEXANDRE, F. Bitcoin, um Sistema inflacionário ou deflacionário? MoneyTimes, 2020. Disponível em: < https://www.moneytimes.com.br/fabricio-alexandre-bitcoin-um-sistemainflacionario-ou-deflacionario/ > Acesso em 10 de setembro de 2021.

ALVES, P. Desenvolvedores desligam Solana para solucionar apagão de 16 horas. InfoMoney, 2021. Disponível em: https://www.infomoney.com.br/mercados/desenvolvedores-desligam-solana-para-solucionarapagao-de-16-horas/ > Acesso em 19 de setembro de 2021.

ANDRADE, J. EUA, China e Venezuela Confira os países que mais negociam bitcoins. ESTADÃO, 2020. Disponível em: < https://einvestidor.estadao.com.br/investimentos/10paises-bitcoin > Acesso em 14 de setembro de 2021.

ARAÚJO, H. P.; SILVA, R. B. A. R. A Tecnologia Digital Blockchain: Análise Evolutiva e Pragmática. Revista Fatec Zona Sul, 2017, p.6-10.

BARDIN, L. Análise de conteúdo. São Paulo: Edições 70, 2011, 229 p.

BELMIRO, J. N. Blockchain e o Potencial de Novos Modelos de Negócios: Um Mapeamento Sistemático. Revista de Gestão e Projetos, Vol.9, n. 3 set. /dez. 2018

BERTOLUCCI, G. 'Rainha do Bitcoin' está de volta? Suposto vídeo de líder é republicano Youtube. Yahoo!esportes, 2021. Disponível em: < https://esportes.yahoo.com/noticias/rainha-bitcoin-est\%C3\%A1-volta-suposto131818835.html > Acesso em 19 de setembro de 2021.

BORTOLINI, R. Como funciona o Blockchain em 4 passos. Sml.Blog, 2018. Disponível em $<$ https://blog.smlbrasil.com.br/como-funciona-o-blockchain-em-quatro-passos/> Acesso em 29 de abril de 2021.

BRAGA, A. M.; FORMIGONI, J. R.; LEAL, R. L. V.; Tecnologia Blockchain: uma visão geral, 2017.

BRANCO, D. C.; Criptomoeda Litecoin sobe $30 \%$ após divulgação de fake news. Canaltech, 2021. Disponível em: < https://canaltech.com.br/mercado/criptomoeda-litecoin-sobe-30-aposdivulgacao-de-fake-news-195761/ > Acesso em 19 de setembro de 2021.

CAMPOS, G. I. R. V. Bitcoin: Consequências Jurídicas do Desenvolvimento da Moeda Virtual. Revista Brasileira de Direito, 11 (2): 77-84, jul.-dez. 2015.

CARDOSO, B. Contratos inteligentes: descubra o que são e como funcionam. Jusbrasil, 2018. Disponível em: < https://brunonc.jusbrasil.com.br/artigos/569694569/contratosinteligentes-descubra-o-que-sao-e-como-funcionam > Acesso em 08 de agosto de 2021.

CARNEIRO, M. F. S. Gestão Pública: o papel do planejamento estratégico, gerenciamento de portfólio, programas e projetos e os escritórios de projetos na modernização da gestão pública. Rio de Janeiro: Brasport, 2010.

CHIAVENATO, I.; SAPIRO, A. Planejamento Estratégico: fundamentos e aplicações 1. ed. $13^{\mathrm{a}}$ triagem. Rio de Janeiro: Elsevier, 2003. 
CIAIAN, P.; RAJCANIOVA, M.; KANCS, D. The economics of Bitcoin price formation. Applied Economics, v. 48, n. 19, p. 1799-1815, 2016.

CRIPTONIZANDO. IOHK anuncia "Maior Implantação de Blockchain do Mundo" com Cardano na Etiópia. Investing.com, 2021a Disponível em: $<$ https://br.investing.com/news/cryptocurrency-news/iohk-anuncia-maior-implantacao-deblockchain-do-mundo-com-cardano-na-etiopia-859278> Acesso em 30 de abril de 2021.

. Morgan Stanley aumenta a exposição a bitcoins. Investing.com, 2021b. Disponível em: < https://br.investing.com/news/cryptocurrency-news/morgan-stanleyaumenta-exposicao-a-bitcoins-909929 > Acesso em 08 de setembro de 2021.

DA REDAÇÃO. Mineração de Bitcoin já consome mais energia do que a Argentina. ISTO É DINHEIRO, 2021. Disponível em: < https://www.istoedinheiro.com.br/mineracao-debitcoin-ja-consome-mais-energia-do-que-a-argentina/ > Acesso em 07 de Agosto de 2021.

DAYCHOUM, M. 40 Ferramentas e Técnicas de Gerenciamento. $1^{\text {a }}$ Ed. [s.1.] Brasport, 2007. p. 15.

DOW, S. The regional composition of the money multiplier process. Scottish Journal of Political Economy. v. 19, n.1, 1982.

EDELMAN. 2013 Edelman Trust Barometer. Edelman, 2013. Disponível em <https://www.edelman.com/trust/2013-trust-barometer> Acesso em 15 de Abril de 2021. Global Deck. p. 22.

FERREIRA, J. E.; PINTO, F.G.C; SANTOS, S. C. Estudo de Mapeamento Sistemático sobre as tendências e desafios do Blockchain. Revista Gestão. Org, v.15, Edição Especial, 2017. p.108-117.

FIALHO, T. M. M.; JAYME Jr. F. G.; HERMETO, A. M. Desenvolvimento do sistema financeiro e pobreza no Brasil: uma análise multivariada. Economia e Sociedade, Campinas, v. 25, n.1., p. 247-278, abr. 2016.

FINNESETH, J. Volumes de DeFi e DEX disparam em meio à proibição de criptomoedas na China e à regulamentação dos EUA. COINTELEGRAPH, 2021. Disponível em: < https://cointelegraph.com.br/news/defi-and-dex-volumes-soar-amid-china-s-crypto-ban-andongoing-us-regulation > Acesso em 10 de Outubro de 2021.

FREITAS, B. A. Crise financeira de 2008: você sabe o que aconteceu? Politize, 2020 Disponível em: <https://www.politize.com.br/crise-financeira-de2008/\#: :text=O\%20que\%20foi\%20a\%20crise,aumento\%20de\%20renda\%20da\%20popula\% C3\%A7\%C3\%A3o.> Acesso em 13 de Abril de 2021.

HARADA, E. Y. Do escambo ao Bitcoin: a história de como o dinheiro evoluiu. Tecmundo, 2020. Disponível em <https://www.tecmundo.com.br/mercado/176339-escambo-bitcoinhistoria-dinheiro-evoluiu.htm_ Acesso em 15 de Abril de 2021.

HESSEL, R. Inflação alta também é um problema nos Estados Unidos. CORREIO BRAZILIENSE， 2021. Disponível < 
https://www.correiobraziliense.com.br/economia/2021/06/4930538-inflacao-alta-tambem-eum-problema-nos-estados-unidos.html > Acesso em 02 de setembro de 2021.

IGNACIO, B. Dogecoin sobe 200\% em 24 horas após tweet de Elon Musk. Tecnoblog, 2021. Disponível em: < https://tecnoblog.net/433000/dogecoin-sobe-200-em-24-horas-apos-tweetde-elon-musk/ > Acesso em 03 de setembro de 2021.

KALASHNIKOV, H. Quantas vezes a China já baniu o Bitcoin? Livecoins, 2021. Disponível em: < https://livecoins.com.br/quantas-vezes-a-china-ja-baniu-o-bitcoin/ > Acesso em 04 de outubro de 2021.

LIMA, B.; GERBELLI, L. G. Inflação no Brasil é a terceira maior da América Latina, atrás somente de Argentina e Haiti. G1, 2021. Disponível em: < https://g1.globo.com/economia/noticia/2021/09/07/inflacao-no-brasil-e-a-terceira-maior-daamerica-latina-atras-somente-de-argentina-e-haiti.ghtml > Acesso em 02 de setembro de 2021.

MARINHO, G. 22\% de todos os dólares americanos da história foram emitidos em 2020. Cointimes, 2020. Disponível em: < https://cointimes.com.br/22-de-todos-os-dolaresamericanos-da-historia-foram-emitidos-em-2020/ > Acesso em 02 de setembro de 2021.

MATTAR, F. N. Pesquisa de marketing: metodologia, planejamento, execução e análise, $2^{a}$ Edição. São Paulo: Atlas, 1994.

MERCADO BITCOIN. Fan Token: faça parte das iniciativas do seu time internacional preferido. Mercado Bitcoin, 2020. Disponível em: < https://www.mercadobitcoin.com.br/fan-tokens > Acesso em 02 de setembro de 2021.

MESQUITA, G. A guerra do Bitcoin no sistema financeiro e como isso pode te afetar. Santos Bancários, 2021. Disponível em: < https://santosbancarios.com.br/artigo/a-guerra-do-bitcoinno-sistema-financeiro-e-como-isso-pode-te-afetar > Acesso em 08 de agosto de 2021.

MOUGAYAR, W. Blockchain para negócios: Promessa, prática e aplicação da nova tecnologia da Internet. Rio de Janeiro: Alta Books Editora, 2017.

NAKAMOTO, S. Bitcoin: A Peer-to-Peer Electronic Cash System. Bitcoin.org, 2008. p.1-4.

NASCIMENTO, D. P. Polkadot e a internet 3.0. MoneyTimes, 2021. Disponível em: < https://www.moneytimes.com.br/escolacripto-polkadot-e-a-internet-3-0/ > Acesso em 07 de agosto de 2021.

PAYÀ, N. Contaminação por criptomoedas e o grande impacto ambiental. Tempo.com, 2021. Disponível em: < https://www.tempo.com/noticias/actualidade/contaminacao-porcriptomoedas-e-o-grande-impacto-ambiental-clima-poluicao.html $>$ Acesso em 03 de setembro de 2021.

PIRES, T. P. Tecnologia Blockchain e suas Aplicações para o Provimento de Transparência em Transações Eletrônicas. Graduação - Faculdade de Tecnologia, Universidade de Brasília. Distrito Federal, 2016. 
PONTES, J. E. Análise Swot Como Ferramenta De Gestão Estratégica: um Estudo de Caso na Divisão de Segurança da UEPB. Monografia (Especialização em Planejamento e Gestão Pública) - Centro de Ciências Sociais Aplicadas, Universidade Estadual da Paraíba. Paraíba, 2014.

PRADO, J. O que é blockchain? [indo além do Bitcoin]. Tecnoblog, 2018. Disponível em < https://tecnoblog.net/227293/como-funciona-blockchain-bitcoin/ > Acesso em 29 de abril de 2021.

REDAÇÃO. O que é NFT e porque esse mercado vale milhões. Casa Abril, 2021. Disponível em: < https://casa.abril.com.br/arte/o-que-e-nft/ > Acesso em 03 de setembro de 2021.

RUBINSTEINN, G. Para especialistas, recuperação de bitcoins pelo FBI é boa para o mercado. EXAME, 2021b. Disponível em: < https://exame.com/future-ofmoney/cybersecurity/para-especialistas-recuperacao-de-bitcoins-pelo-fbi-e-boa-para-omercado/ > Acesso em 03 de setembro de 2021.

SADOK, M. Acusado de esquema de pirâmide com criptomoedas movimentou R \$ 38 bi em 6 anos. Band, 2021. Disponível em: < https://www.band.uol.com.br/noticias/acusado-deesquema-com-piramide-com-criptomoedas-movimentou-r\$-38-bi-em-6-anos-16368876 > Acesso em 19 de setembro de 2021.

SÉRVIO, G. Bitcoin é banido na China; país proíbe todas as transações em criptomoedas. OLHAR DIGITAL, 2021. Disponível em: https://olhardigital.com.br/2021/09/24/pro/bitcoin-banido-china-criptomoedas/ > Acesso em 04 de outubro de 2021.

SICHEL, R. L.; CALIXTO, S. R. Criptomoedas: Impactos na Economia Global. Perspectivas. Revista de Direito da Cidade, vol. 10, nº3, 2018.

SILVA, V. A. F.; BOVÉRIO, M. A. Blockchain: uma tecnologia além da criptomoeda virtual. Interface Tecnológica, vol. 15, n.1, 2018.

SILVA, G. A. B; RODRIGUES, C. K. S. Rentabilidade econômica da mineração de bitcoins e litecoins. Universitas. Gestão e Tecnologia. 2017.

SIMÕES, L. F. Irã bane a mineração de criptomoedas para evitar apagões no verão. ESTADÃO, 2021. Disponível em: < https://einvestidor.estadao.com.br/noticias/ira-banemineracao-de-criptomoedas-temporariamente > Acesso em 03 de setembro de 2021.

SOBRINHO, R. P.; GARCIA J.R.; MAIA A.G.; ROMEIRO A.R. Tecnologia Blockchain: inovação em Pagamentos por Serviços Ambientais. Revista Brasileira de Inovação, v. 18, n. 1, p. 157-176, 2019.

SOUSA, R. Bitcoin é imune à inflação? Especialistas explicam como moeda pode ser usada para escapar do dragão. SEUDINHEIRO, 2021. Disponível em: < https://www.seudinheiro.com/2021/economia/bitcoin-criptomoeda-inflacao-07-07/ > Acesso em 08 de agosto de 2021. 
STEIN, L. Lista mostra 11 países que baniram criptomoedas; conheça. BEINCRYPTO, 2021. Disponível em: < https://beincrypto.com.br/lista-mostra-11-paises-que-baniramcriptomoedas-conheca/> Acesso em 08 de agosto de 2021.

SWAN, M. Blockchain: Blueprint for a New Economy. Cambridge: O’ Reilly, 2015.

THE BLOCK. Presidente Putin assina lei que proíbe o uso de criptoativos para pagamentos no país. CryptoTimes, 2020. Disponível em: < https://www.moneytimes.com.br/presidenteputin-assina-lei-que-proibe-o-uso-de-criptoativos-para-pagamentos-no-pais/>Acesso em 16 de abril de 2021.

THE NEW YORK TIMES. Why Bill Gates Is Worried About Bitcoin. The New York Times, 2021. Disponível em: <https://www.nytimes.com/2021/03/09/business/dealbook/billgates-bitcoin.html > Acesso em 09 de setembro de 2021.

TIDY, J. Hacker que desviou R 33 bilhões em criptomoedas devolve quase tudo e explica ação. BBC NEWS, 2021. Disponível em: < https://www.bbc.com/portuguese/internacional$58200536>$ Acesso em 03 de setembro de 2021.

TRADINGVIEW. Bitcoin/DólarAmericano 2021. Disponível em <https://br.tradingview.com/symbols/BTCUSD/> Acesso em 29 de abril de 2021.

ULRICH, F. Bitcoin: a moeda na era digital. Instituto Ludwig von Mises Brasil, São Paulo, 2014.

VARONI, M. Atualizações na Bitcoin: o que são e como acontecem os forks. Techtudo, 2017. Disponível em: < https://www.techtudo.com.br/noticias/2017/12/atualizacoes-nabitcoin-o-que-sao-e-como-acontecem-os-forks.ghtml > Acesso em 03 de setembro de 2021.

VERGARA, S. C. Projetos e Relatórios de Pesquisa em Administração. São Paulo: Atlas, 1998.

\section{Como Referenciar este Artigo, conforme ABNT:}

BRAGA JÚNIOR, ASRILHANT, P. G. B. Análise do Mercado de Criptomoedas Sob o Enfoque da Matriz Swot. Rev. FSA, Teresina, v.18, n. 11, art. 3, p. 44-67, nov. 2021.

\begin{tabular}{|l|c|c|}
\hline \multicolumn{1}{|c|}{ Contribuição dos Autores } & $\begin{array}{c}\text { P. G. Braga } \\
\text { Júnior }\end{array}$ & $\begin{array}{c}\text { B. } \\
\text { Asrilhant }\end{array}$ \\
\hline 1) concepção e planejamento. & $X$ & $X$ \\
\hline 2) análise e interpretação dos dados. & $X$ & $X$ \\
\hline 3) elaboração do rascunho ou na revisão crítica do conteúdo. & $X$ & $X$ \\
\hline 4) participação na aprovação da versão final do manuscrito. & & $X$ \\
\hline
\end{tabular}

